\title{
Factores de riesgo en adolescentes infractores del Centro Juvenil El Tambo
}

\author{
Luchkanap kayninkuna huchapakuśha \\ walaśhwamlakunaćhu maltakunap \\ wichaśhawasi Tampukaćhu (El Tambo)
}

\author{
Kantagetari ira iboingapage \\ amasitagekengicha inaigiro kara tampokë
}

Recibido: 17 marzo 2020 Corregido: 22 abril 2020 Aprobado: 19 mayo 2020

Yovana Luiza Alberto Bueno Nacionalidad: Peruana Filiación: Universidad Nacional del Centro del Perú Correo:yalberto@uncp.edu.pe ORCID: https://orcid.org/0000-0003-1207-9273

\section{Resumen}

Investigación básica de naturaleza descriptiva, población 50 adolescentes infractores del programa intermedio del centro juvenil de el Tambo; los factores de riesgo medio ambiental social en adolescentes en lo personal presentó dificultades negativas en actitudes y comportamientos, baja autoestima, carencia de valores; en lo escolar inasistencia al colegio, evasiones, bajas calificaciones, repitencia escolar, conflictos con sus profesores, incumpliendo de tareas académicas, abandono escolar; en lo familiar antecedentes de padres recluidos, haber recibido castigos físicos y psicológicos, evidenciar situaciones de violencia, carencia de afectividad, abandono moral y material. Conclusión: factores personales, social y familiar.

\section{Palabras clave:}

Factores personales, escolar, familiar.

\section{Lisichiku limaykuna:}

Nunakayp kayninkuna, yaćhapaku, ayllup.

\section{Nibarintsipage katingatsaro:}

kantagetari paniropage, sangenaitatsi,itimaigirori-

Datos de la autora

Yovana Luiza Alberto Bueno. Investigador y docente en proyectos sociales. Magister en administración. 


\section{Risk Factors in Teenagers Offenders of the Juvenile Detention Center of El Tambo}

\begin{abstract}
Basic research of a descriptive nature, population of 50 teenagers who violate the intermediate program of the Juvenile Detention Center of El Tambo; the factors of social environmental risk in adolescents personally presented negative difficulties in attitudes and behaviors, low self-esteem, lack of values; in school, non-attendance at school, evasions, low grades, school repetition, conflicts with their teachers, failing to fulfill academic tasks, dropping out of school; in the family history of confined parents, having received physical and psychological punishments, evidencing situations of violence, lack of affectivity, moral and material abandonment. Conclusion personal, social and family factors.
\end{abstract}

\section{Keywords}

Personal, school, family

factors.

\section{Fatores de risco em adolescentes infratores do Centro Juvenil El Tambo}

\section{Resumo}

Pesquisa básica de natureza descritiva, população de 50 adolescentes infratores do programa intermediário do centro juvenil de El Tambo; os fatores de risco mediano socioambiental em adolescentes apresentaram pessoalmente dificuldades negativas em atitudes e comportamentos, baixa autoestima, carência de valores; no escolar: não frequência à escola, evasões, notas baixas, repetição escolar, conflitos com seus professores, não cumprimento das tarefas acadêmicas, abandono escolar; no familiar: antecedentes de pais reclusos, ter recebido castigos físicos e psicológicos, evidencias de situações de violência, carência de afetividade, abandono moral e material. Conclusão: fatores pessoais, sociais e familiares.

\section{Palavras-chave:}

Fatores pessoais, escolar, familiar 


\section{Introducción}

La adolescencia es un proceso donde el ser humano se encuentra en una etapa de transición de la niñez a una etapa adulta. Es una etapa del ciclo vital en las que a una mayor capacidad intelectual se suma una mayor independencia; los adolescentes y jóvenes hacen en el tiempo en que no están con sus padres ni bajo la supervisión de los adultos es variable (López y Rodríguez, 2012, p26).

Estas tres etapas sucesivas, prepubescencia, pubertad y adolescencia, en muchos casos pasan inadvertidas, por eso es importante conocer sus características a fin de entender el comportamiento de los estudiantes, este desconocimiento hace que se adopten decisiones apresuradas, generalmente punitivas que solo generan un mayor conflicto y complican su relación con el medio familiar, escolar y social. Estos cambios es motivo de complacencia en algunos, otros se sienten avergonzados. Según sea la acogida o intolerancia que experimenten. Cuando las familias son disfuncionales, el maltrato lleva a otras dificultades, cuando son expulsados prematuramente de la familia, prefieren incorporarse en las pandillas donde gozan de la seguridad que no la encuentran en otros contextos.

En la infancia y la adolescencia, resulta habitual que comportamientos antisociales e incluso delictivos se correspondan a una conducta normal del niño y adolescente, formando parte del proceso de crecimiento, aprendizaje y desarrollo social de los mismos, la mayor parte de esta delincuencia es de carácter leve, episódica y no suele dejar posteriores efectos negativos. Por otra parte, señalan que los niños y adolescentes que cometieron delitos más graves y frecuentes, tienen más posibilidades de convertirse en delincuentes habituales. La mayoría de los delincuentes crónicos, de carrera o multi reincidentes empezaron su actividad criminal a edades tempranas (infancia y adolescencia), se ha prestado una mayor atención a los déficits de desarrollo de la personalidad y a los vínculos sociales formados durante la infancia como precursores de una posterior conducta antisocial y delictiva (Vásquez, 2015; p.2).

El comportamiento delincuencial en adolescentes se presenta por su inexperiencia, carencia de madurez y una débil formación de su personalidad, en muchos de los casos exponiéndose a situaciones difíciles, comprometedoras y muy peligrosas para su vida. En el estudio análisis de los factores de riesgo que influyen en los adolescentes infractores de la ley penal en Girardot de Sánchez y Velásquez (2016) señala “... edades más propensas que delinquen son entre 15 a 17 años, los factores están ligados a las correlaciones directas con mayor probabilidad es la baja autoestima y falta de comunicación; ser miembro de una banda delincuencial y baja autoestima".

Según los reportes del Ministerio de Justicia en los últimos años se ha percibido un incremento en la participación de adolescentes en la comisión de delitos o faltas; que por su corta edad y en amparo a la normatividad no permite a la justicia sancionar por la gravedad de los ilícitos. Salas, Martínez, Matos et al (2017; p.12) citado INDAGA (2016) señala “... que los adolescentes son inimputables frente al sistema de justicia penal, son responsables frente al sistema de administración de justicia especializada, el cual responde a su estado de desarrollo en el marco del ciclo de vida de los adolescentes". Este sistema debe poseer una orientación socioeducativa, en lugar de concentrase en la aplicación de penas y debe buscar como fin la reinserción del adolescente dentro de la sociedad Salas, Martínez, Matos et al (2017; p.12) citado INDAGA (2016). 
Según el Instituto Nacional de Estadística e Informática (2015) estima “... para el 2017 la población adolescente de 12 a 17 años, representará el 10.9\% del total de la población en el Perú; sin embargo, el crecimiento anual de los adolescentes irá disminuyendo hasta alcanzar al 10\% para el 2025". Para poder aproximarnos al fenómeno de la delincuencia juvenil, es preciso aclarar el sistema de justicia juvenil de nuestro país, hace una distinción entre menores y mayores considerando persona adulta a partir de los 18 años de edad y puede ser sometido a todos los procesos judiciales y sanciones penales que correspondan; si la persona tiene una

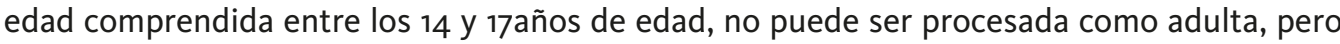
es sometida a un proceso especial.

Así mismo, un estudio sobre adolescentes infractores, elaborado por el Observatorio Nacional de Política Criminal INDAGA (2017) señala que "el sistema de justicia juvenil debe incidir en aquellos factores que originan el tipo de conducta y las circunstancias que llevaron a los adolescentes a cometer la infracción".

En los últimos años, el aumento significativo de la inseguridad ciudadana y su vinculación con el incremento de la participación de adolescentes en hechos violentos constituye uno de los problemas más importantes que aquejan al país.

Durante el periodo 2011-2016, el número de adolescentes en conflicto con la ley penal atendidos por el SRSALP se ha ido incrementando de 4,736 adolescentes atendidos en el 2011 a 6,950 adolescentes atendidos en el 2016 en ambos modelos de tratamiento. Este aumento guarda relación con el número de adolescentes involucrados en infracciones a la ley penal que registra la Policía Nacional del Perú - PNP que pasó de 4,334 en el 2011 a 4,618 hechos en el 2016 (Ministerio de Justicia y Derechos Humanos, 2017) citado en (Sierra; 2019 p.2).

Los adolescentes que se encuentran recluidos presentan una mayor frecuencia de exposición a los factores de riesgo, asociados con la conducta antisocial y delictiva, en comparación con los adolescentes no infractores que asisten a una institución pública, en los niveles exosistema, microsistema y macrosistema. Los dos grupos de adolescentes se encuentran expuestos al maltrato, el consumo y abuso de alcohol en proporciones similares constituyen los factores de riesgo que más se asocian a la generación del comportamiento antisocial y el comportamiento delictivo Sanabria \& Uribe (2010).

El objetivo fue conocer los factores de riesgo que presentaron los adolescentes infractores del centro juvenil del El Tambo, el estudio busca contribuir en los niveles preventivos a nivel familiar e institucional, referidos al fortalecimiento de capacidades y actitudes en la educación básica regular tanto en instituciones públicas y privadas.

\section{Material y Método}

La investigación fue de tipo básica, nivel y carácter descriptivo, método general y científico, como unidad de análisis los adolescentes infractores del Centro Juvenil de El Tambo, con una población y muestra de 50 internos del programa intermedio, con una probabilidad al $95 \%$, el instrumento que se utilizó fue el cuestionario escala y para la confiabilidad del instrumento se utilizó el alfa de Cronbach con una aprobación del o,683. 


\section{Resultados}

Tabla 1

Datos Generales

\begin{tabular}{|c|c|c|}
\hline CARACTERISTICAS Y CATEGORÍAS & FRECUENCIA & PORCENTAJE \% \\
\hline $\begin{array}{l}\text { Edad } \\
\text { Menor de } 15 \text { años } \\
16 \text { años } \\
17 \text { años } \\
\text { Mas de } 18 \text { años }\end{array}$ & $\begin{array}{l}13 \\
13 \\
13 \\
11\end{array}$ & $\begin{array}{l}26,0 \\
26,0 \\
26,0 \\
22,0\end{array}$ \\
\hline $\begin{array}{l}\text { Procedencia } \\
\text { Huancayo } \\
\text { Satipo } \\
\text { Concepción } \\
\text { Jauja } \\
\text { Chupaca } \\
\text { Chanchamayo } \\
\text { Tarma } \\
\text { Junín } \\
\text { Otro }\end{array}$ & $\begin{array}{c}6 \\
8 \\
5 \\
10 \\
3 \\
3 \\
4 \\
2 \\
9\end{array}$ & $\begin{array}{c}12,0 \\
16,0 \\
10,0 \\
20,0 \\
6,0 \\
6,0 \\
8,0 \\
4,0 \\
18,0\end{array}$ \\
\hline $\begin{array}{l}\text { Motivo por lo que está internado } \\
\text { Violación } \\
\text { Robo } \\
\text { Homicidio } \\
\text { Otro }\end{array}$ & $\begin{array}{c}20 \\
28 \\
1 \\
1\end{array}$ & $\begin{array}{l}40,0 \\
56,0 \\
2,0 \\
2,0\end{array}$ \\
\hline $\begin{array}{l}\text { Tiempo que está internado en el centro juvenil } \\
\text { Menos de } 1 \text { año } \\
1 \text { a } 2 \text { años } \\
3 \text { a } 4 \text { años }\end{array}$ & $\begin{array}{l}19 \\
21 \\
10\end{array}$ & $\begin{array}{l}38,0 \\
42,0 \\
20,0\end{array}$ \\
\hline $\begin{array}{l}\text { Numero de veces que estuvo en el Centro Juve } \\
\text { Primera vez } \\
2 \text { veces }\end{array}$ & $\begin{array}{l}43 \\
7\end{array}$ & $\begin{array}{l}86,0 \\
14,0\end{array}$ \\
\hline
\end{tabular}

Fuente: Los datos son obtenidos por la investigadora 2019.

En la tabla 1, se observa las características generales de los adolescentes infractores internado en el Centro Juvenil de El Tambo del programa intermedio; donde el 26,0\% son menores de 15 años de edad, de 16 y 17 años de edad es el mismo porcentaje; así mismo albergan jóvenes mayores de 18 años de edad en un 22,0\% quienes están cumpliendo con las medidas correctivas dictadas por el Ministerio de Justicia. Con respecto a la procedencia; el 20,0\% de los adolescentes infractores proceden de la provincia de Jauja, seguida por Satipo con un 16,0\%, Huancayo con un 12,0\%, Concepción 10,0\%, Tarma con un 8,0\%, la Provincia de Chupaca, Chanchamayo con un $6 \%$, la provincia de Junín con un $4 \%$ y con un $18 \%$ de adolescentes que proceden de otros departamentos del país. Los motivos por lo que están internados los adolescentes infractores son: el 56,0\% manifiestan que es por robo, seguida por el 40,0\% que manifiestan que están por motivo de violación, el 2\% por homicidio y otros motivos. Con respecto a los números de veces que han sido internados ellos manifiestan que es por primera vez un $86,0 \%$ y por segunda vez un $14,0 \%$. 
Tabla 2

Factor de riesgo medio ambiental social: personal o Individual

\begin{tabular}{|l|c|c|c|}
\multicolumn{1}{c}{ FACTORES O ITEMS } & \multicolumn{1}{c}{ RESPUESTA (\%) } \\
\hline Crees que tus actitudes son adecuadas para tu edad & Siempre & A veces & Nunca \\
\hline Has tenido problemas por tus actitudes & 6,0 & 96,0 & 0 \\
\hline $\begin{array}{l}\text { Has peleado con tus compañeros o amigos por algún } \\
\text { amigo }\end{array}$ & 8,0 & 88,0 & 6,0 \\
\hline $\begin{array}{l}\text { Discutes con tus compañeros o amigos } \\
\text { Respetas las decisiones u opiniones de los demás }\end{array}$ & 10,0 & 78,0 & 14,0 \\
\hline $\begin{array}{l}\text { Cuando una persona necesita de tu ayuda le prestas } \\
\text { atención }\end{array}$ & 12,0 & 56,0 & 34,0 \\
\hline $\begin{array}{l}\text { Intervienes para resolver los problemas, cuando tus } \\
\text { amigos o compañeros están peleando o discutiendo }\end{array}$ & 10,0 & 66,0 & 24,0 \\
\hline Tienes dificultades para resolver tus problemas & 20,0 & 64,0 & 24,0 \\
\hline
\end{tabular}

Fuente: Idem.

En la tabla 2, observamos resultados del factor de riesgo medio ambiental social en lo personal, de los adolescentes infractores del Centro Juvenil de El Tambo, el 96,0\% "a veces" las actitudes de los adolescentes son adecuadas para su edad y el 4,0\% "siempre" sus actitudes son adecuadas; a la pregunta ¿has tenido problemas por tus actitudes? el 88,0\% "a veces" han tenido problemas, el 6,0\% manifestaron que "siempre" y el 6,0\% nunca; si han peleado con sus compañeros o amigos por algún motivo el 78,0\% manifestaron que "a veces", el 8,0\% "siempre" y un 6,0\% "nunca"; si ¿discutes con tus compañeros y amigos? Respondieron el 56,0\% "siempre", el 34,0\% nunca y el 10,0\% "siempre".

Referido al respeto de las opiniones de los demás, el 66,0\% “a veces", el 24,0\% "nunca" y el 10,0\% "siempre"; cuando una persona necesita de tu ayuda le prestas atención el $64,0 \%$ de los adolescentes respondieron "a veces" le presta atención, el 24,0\% "nunca" y el 12,0\% "siempre" está atento para servir a su prójimo; si Ud. Interviene para resolver problemas de sus amistades o compañeros cuando están peleando o discutiendo el 62,0\% "a veces" intervienen, el 28,0\% "nunca" interviene frente a estos hechos y el 10,0\% "siempre" están atentos para ayudarlos; si han tenido dificultades para resolver sus problemas el 52,0\% "a veces", el $28,0 \%$ "nunca" y el "20,0\% "siempre" se han presentado dificultades para resolverlos.

Tabla 3

Factor de riesgo medio ambiental social: Escolar

\begin{tabular}{|l|c|c|c|} 
& \multicolumn{3}{c}{ RESPUESTA (\%) } \\
\hline \multicolumn{1}{|c|}{ FACTORES O ITEMS } & Siempre & A veces & Nunca \\
\hline Te sientes bien con las decisiones que tomas para tu vida & 42,0 & 50,0 & 8,0 \\
\hline $\begin{array}{l}\text { Antes de ingresar al Centro Juvenil, asistías regularmente } \\
\text { al colegio }\end{array}$ & 30,0 & 64,0 & 6,0 \\
\hline Has registrado faltas en el colegio & 26,0 & 66,0 & 8,0 \\
\hline Has tenido problemas con algún compañero del colegio & 34,0 & 52,0 & 14,0 \\
\hline $\begin{array}{l}\text { Tus compañeros del colegio te hacían sentir parte del } \\
\text { grupo }\end{array}$ & 24,0 & 60,0 & 16,0 \\
\hline En el colegio tenias amigos de tu confianza & 28,0 & 56,0 & 14,0 \\
\hline
\end{tabular}




\begin{tabular}{|l|l|l|c|}
\hline $\begin{array}{l}\text { Participabas en actividades sociales que el colegio } \\
\text { organizó }\end{array}$ & 28,0 & 66,0 & 6,0 \\
\hline Cumplías con las normas establecidas en el colegio & 38,0 & 56,0 & 6,0 \\
\hline Has sido sancionado por algún motivo en el colegio & 34,0 & 56,0 & 10,0 \\
\hline Cumpliste con las tareas académicas del colegio & 32,0 & 62,0 & 6,0 \\
\hline Has tenido problemas con algún profesor(a) del colegio & 28,0 & 60,0 & 12,0 \\
\hline Te has evadido del colegio por algún motivo & 26,0 & 66,0 & 8,0 \\
\hline Has repetido de grado en el colegio & 30,0 & 56,0 & 14,0 \\
\hline Has abandonado el colegio por algún motivo & 32,0 & 56,0 & 12,0 \\
\hline Has desaprobado alguna asignatura en el colegio & 32,0 & 62,0 & 4,0 \\
\hline Has consumido licor o drogas en alguna ocasión & 44,0 & 42,0 & 14,0 \\
\hline
\end{tabular}

En la tabla 3, observamos datos del factor de riesgo medio ambiental social en lo escolar de los internos del Centro Juvenil de El Tambo; los resultados muestran que el 50,0\% respondieron "a veces" se sienten bien con las decisiones que toman para su vida y el 42,0\% "siempre"; con respecto a la asistencia regular a sus colegios el $64,0 \%$ respondieron que "a veces", el $30,0 \%$ "Siempre"; registro de faltas en su institución educativa $66,0 \%$ respondieron que "a veces" registraban faltas y el $26,0 \%$ "siempre"; han presentado problemas con sus compañeros del colegio el 52,0\% respondieron "a veces" y el 34,0\% "siempre"; si sus compañeros le hacían sentir parte del grupo, el 60,0\% respondieron "a veces" y el 24,0\% "siempre”; si tenían amigos de su confianza en el colegio, el 56,0\% "a veces" y el 28,0\% "siempre"; si participaban en las actividades que organizaba el colegio el $66,0 \%$ respondieron "a veces" y el $28,0 \%$ "siempre".

Con respecto a las normas establecidas por el colegio respondieron; el 56,0\% "a veces" cumplían con las normas y el 38,8\% "siempre"; si han sido sancionado por algún motivo en el colegio el 56,0\% respondieron "a veces" y el 34,0\% "siempre"; si cumplían tareas académicas del colegio, el 62,0\% de los adolescentes infractores respondieron que "a veces" y el 32,0\% "siempre"; si han tenido problemas con algún profesor (a) el 60,0\% respondieron que "a veces" y el $28,0 \%$ respondieron que "siempre"; si se han evadido del colegio por algún motivo el 66,0\% de los adolescentes respondieron que "a veces" y el $26,0 \%$ respondieron que "siempre"; si han abandonado el colegio por algún motivo el 56,0\% manifestaron que "a veces" y el 32,0 "siempre", si han repetido de grado en el colegio, el 56,0\% "a veces" y el 30,0\% "siempre"; si han consumido licor o drogas, el $42,0 \%$ respondieron "a veces" y el $44,0 \%$ "siempre" han consumido.

\section{Tabla 4}

\section{Factor de riesgo medio ambiental social: Familiar}

\begin{tabular}{|l|c|c|c|}
\hline \multicolumn{1}{|c}{ FACTORES O ITEMS } & \multicolumn{3}{c|}{ RESPUESTA (\%) } \\
\hline Tus padres alguna vez ha sido detenido o recluido & Siempre & A veces & Nunca \\
\hline Tus padres te castigaban cuando eras niño & 10,0 & 64,0 & 26,0 \\
\hline Has visto peleas de tus padres & 22,0 & 56,0 & 22,0 \\
\hline Tus padreste aconsejan para que tengas buencomportamiento & 24,0 & 60,0 & 16,0 \\
\hline Tus padres te corrigen cuando te portas mal & 10,0 & 76,0 & 14,0 \\
\hline Recibes afecto, cariño y palabras de aliento por parte de tus & 8,0 & 76,0 & 16,0 \\
\hline \begin{tabular}{l|l} 
padres \\
Tus padres aportan a la economía del hogar
\end{tabular} & 6,0 & 78,0 & 16,0 \\
\hline En tu familia, cuentas con la economía necesaria para & 6,0 & 74,0 & 20,0 \\
\hline solventar tus gastos & 4,0 & 64,0 & 32,0 \\
\hline
\end{tabular}


En la tabla 4, observamos datos del factor de riesgo medio ambiental social en lo familiar, donde los resultados nos muestran lo siguiente: si alguna vez tus padres han sido detenidos o recluidos, el 64,0\% respondieron que "a veces" y el 10,0\% siempre han sido detenidos por la policía o recluidos al algún establecimiento penitenciario; si recibiste castigos por parte de tus padres cuando fuiste niño, el 56,0\% "a veces" y el 22,0\% "siempre"; si han sido testigos de la pelea de sus padres, el $60,0 \%$ de los adolescentes respondieron "a veces" y el $24,0 \%$ "siempre"; si recibiste recomendaciones y/o consejos por parte de tus padres, el $76,0 \%$ respondieron que "a veces" y el 14\% "nunca", si tus padres te corrigieron cuando te portaste mal, el 76,0\% "a veces" y el 16,0\% "nunca"; si recibiste afecto, cariño y palabras de aliento por parte de tus padres, el 78,0\% respondieron que "a veces" y el 16,0\% "nunca", si tus padres aportan a la economía del hogar para cubrir gastos de alimentación, educación, salud entre otros, el 74,0\% "a veces" y el 20,0\% respondieron que "nunca"; si tus padres cuentan con la economía necesaria para solventar sus gastos de alimentación, educación, salud y otros el " $64,0 \%$ "a veces" cuentan con los recursos y el 32,0\% respondieron que "nunca" cuentan con los recursos para solventar gastos del hogar.

\section{Discusión}

HE1. El factor de riesgo medio ambiental social en lo personal influenciaron en los adolescentes infractores del Centro Juvenil El Tambo, porque el 96,0\% respondieron que "a veces" sus actitudes son adecuadas para su edad, el $88,0 \%$ "a veces" han tenido problemas por sus actitudes, el $78,0 \%$ de ellos "a veces" han peleado con amigos o compañeros por algún motivo, el $56,0 \%$ "a veces" discuten con sus compañeros hechos que evidencian la conductas poco tolerables, el $66,0 \%$ de ellos "a veces" respetan las opiniones o decisiones de los demás, con respecto a la solidaridad el "64,0\%" "a veces" apoyan cuando alguna persona lo necesita; con respecto a la dificultad para resolver problemas el 52,0\% "a veces" tienen estas dificultades y el 50,0\% "a veces" se sienten bien con las decisiones que toman para su vida, con estos hechos se puede evidencias que muchos de ellos han sufrido agresiones por sus compañeros en alguna oportunidad y presentan una débil autoestima.

Estos datos se sustentan en Sanabria y Uribe (2010) cuando dice que “...los adolescentes se encuentran expuestos al maltrato, el consumo y abuso de alcohol en proporciones similares, constituyen los factores de riesgo que más se asocian a la generación del comportamiento antisocial y el comportamiento delictivo", Redondo et al (2011) señala “...la falta de compromiso genuino y la educación, el déficit de aspiraciones, el déficit de empatía, la dificultad para demorar la gratificación, las creencias, el déficit de razonamiento moral, una emocionalidad negativa y una baja autoestima serian características que se relacionan a una conducta anti social", por otra parte Flores (2018) señala “...existen factores condicionantes de la conducta juvenil del infractor, los cuales son proclives a presentar conductas antisociales en su relación con sus pares y entorno".

La teoría de la sub cultura delincuente de Cohen (1995) citado en Sánchez y Velásquez (2016) La mayoría de los problemas de adaptación se solucionan de forma normal, pero en algunos casos, las personas eligen soluciones desviadas. Las personas seleccionan, en un primer momento, las soluciones que son compatibles con las expectativas de sus grupos de referencia, pero cuando no se encuentran estas soluciones, se buscan otros grupos cuya cultura proporcione respuestas adecuadas. Ello daría origen a la subcultura, que emerge cuando un número de personas con similares problemas de adaptación no encuentra soluciones institucionalizada, entre ellos tenemos: Incorporarse al ámbito cultural de los jóvenes de clase me- 
dia, aunque suponga competir en inferioridad de condiciones, integrarse a la cultura de otros jóvenes de la calle renunciando a sus aspiraciones o integrarse en una sub cultura delincuente.

HE2. El factor de riesgo medio ambiental social en lo escolar de los adolescentes infractores del Centro Juvenil El Tambo, porque el 64,0\% "a veces" asistía regularmente al colegio, el $66,0 \%$ "a veces" han registrado faltas en el colegio, el 34,0\% "siempre" ha tenido problemas con sus compañeros del colegio, el 56,0\%, el 66,0\% "a veces" participaba en actividades del colegio, el 56,0\% "a veces" cumplían con las normas establecidas por el colegio, el 56,0\% "a veces" han sido sancionados por algún motivo, el 62,0\% "a veces" cumplían con las tareas académicas, el 60,0\% "a veces" han tendido problemas con sus profesores, el 66,0\% se han evadido del colegio, el 30,0\% "siempre" han repetido de grado, el 32,0\% respondieron abandonaron el colegio, el 62,0\% han desaprobado alguna asignatura y el $44,0 \%$ "siempre" han consumido licor o drogas en alguna ocasión.

Estos datos se sustentan en Flores (2018) cuando menciona “...existen factores condicionantes de la conducta juvenil del infractor y son proclives a presentar conductas antisociales en su relación con sus pares y entorno, también se perciben que existen factores que inciden en la conducta, como son lo económico, socio familiar, cultural"; por otra parte Valariño, Amado, Alves (2013) un estudio señala "...los primeros pasos de la carrera delictiva se inician a edades tempranas cuando muestra los resultados de los menores infractores que presentaban problemas de socialización, déficits en inteligencia emocional y tendían a emplear estrategias de afrontamiento improductivas”, López y Rodriguez (2012) señala “...Los factores de riesgo que son predictores comunes de la conducta antisocial y el consumo de sustancias son el fracaso escolar, la implicación en bandas, las actitudes favorables y la iniciación a la conducta antisocial".

La Teoría integradora de Farrington (1992) señala que la falta de recursos económicos, un bajo coeficiente intelectual y una crianza de poca calidad serán los factores de mayor riesgo para el comienzo de la delincuencia. Padres, hermanos antisociales y amigos delincuentes, tendrán una influencia en la continuidad de esas actividades delictivas. Dos importantes conclusiones se pueden extraer del modelo teórico de Farrington, en primer lugar, ha demostrado fehacientemente la continuidad en la delincuencia y los comportamientos criminales, identificando y verificando, en segundo lugar, algunos de los factores predictores de la delincuencia a diferentes edades.

HE3. El factor de riesgo medio ambiental social en lo familiar presentan los adolescentes infractores del Centro Juvenil El Tambo, porque el 64,0\% "a veces" sus padres han sido detenidos o recluidos en algún establecimiento penitenciario; el 56,0\% respondieron que "a veces" sus padres le castigaron cuando eran niños; el 60,0\% de ellos han visto "a veces" las peleas de sus padres; el 76,0\% "a veces" sus padres le aconsejan para que tengan un buen comportamiento; el 78,0\% de los adolescentes reciben cariño y palabras de aliento por parte de sus padres, el 74,0\% "a veces" aportan a la economía del hogar y el 64,0\% "a veces" cuentan con la economía necesaria para solventar los gastos del hogar.

Estos datos se sustentan con Barraza (2009) cuando dice “...la familia juega un papel relevante en el proceso de socialización de los jóvenes”, Redondo et al (2011) señala “...a falta de supervisión, cambios de estructura familiar, malos tratos, crianza punitiva o inadecuada, padres delincuentes, alcohólicos o drogadictos, la falta de comunicación o carencias afectivas, bajo ingresos familiares, etc. Son factores de riesgo que incrementan el riesgo en comportamientos delictivos", también frías et al (2013) señala “...la interacción pobre entre padres e 
hijos y los conflictos maritales son considerados como factores de riesgo familiares para la conducta anti social y delictiva”.

La Teoría del modelo ecológico de Bronfenbrenner (1987-1994) citado en Frías et al (2013) Propone los niveles denominados microsistema, exosistema, macrosistema y mesosistema. El microsistema es el nivel más inmediato en el que se desarrolla el ser humano, comprende la familia, y las dinámicas que al interior de ella suceden; el exosistema lo comprenden contextos más próximos al sistema familiar, incluye instancias como la escuela, la iglesia, las instituciones recreativas y los organismos de control social; el mesosistema comprende las interrelaciones de dos o más entornos en los que se encuentra inmersa la persona; y el macrosistema, conformado por la cultura, las creencias y actitudes de cada persona y los individuos de su sociedad. Estos niveles dependen uno del otro y, por tanto, se requiere de una participación conjunta de los diferentes contextos y de una comunicación entre ellos.

La Teoría integradora de Farrington (1992) citado en Vásquez (2015) señala que la delincuencia se produce mediante un proceso de interacción (dividido en cinco etapas) entre el individuo y el ambiente, llegando a la conclusión, tras contrastar su teoría con los resultados obtenidos por el London Longitudinal projec, que los jóvenes pertenecientes a familias de clase baja tendrán una mayor propensión a la delincuencia, ante su imposibilidad de alcázar legalmente sus metas y objetivos. Los niños maltratados tendrán más probabilidades de delinquir al no haber adquirido controles internos sobre comportamientos desaprobados socialmente, y los niños con amigos y/o familia delincuente tenderán a desarrollar y a justificar actitudes antisociales.

HG. Los factores de riesgo medio ambiental social que presentan los adolescentes infractores del Centro Juvenil de El Tambo, son los personales o individuales porque presentan dificultades en sus actitudes, comportamientos, presentan baja autoestima, carencia de valores; en el factor escolar los adolescentes no asistían regularmente al colegio, presentaban evasiones, bajas notas, repitencia, problemas con sus profesores, incumpliendo de tareas académicas y por ende abandono al colegio y en el factor familiar algunas veces sus padres han sido detenidos o recluidos, recibieron castigos físicos y psicológicos de niños, evidenciaban situaciones de violencia en el hogar, reciben poco afecto, abandono moral y material en el hogar.

La Teoría del desarrollo social de Catalano y Hawkins (1996) citado en Vasquéz (2015) El comportamiento de los jóvenes se encuentra muy influenciado por los vínculos que desarroIlan con los grupos sociales más importantes en sus vidas (familia, escuela, grupo de amigos y comunidad), dependiendo que estos vínculos su futuro comportamiento (prosocial o antisocial). Este modelo teórico pretende averiguar cómo los factores de riesgo y los factores protectores interactúan e influyen en las diferentes etapas del proceso evolutivo de los jóvenes, para alcanzar un desarrollo social o antisocial.

Teoría del aprendizaje social de Ronal Akers 1997 (citado en Redondo, 2008) En este se dice que el comportamiento antisocial y delictivo se aprende a partir de cuatro mecanismos interrelacionados: La asociación diferencias, las definiciones, el reforzamiento diferencial y la imitación de modelos. La asociación diferencial es aquel proceso mediante en cual un individuo es expuesto a definiciones normativas favorables o desfavorables a la conducta antisocial se pueden producir tanto en grupos primarios - familias o amigos - como en el marco de grupos secundarios - vecinos, iglesias, profesores, figuras de autoridad o medios de comunicación; las definiciones hacen referencia a los propios significados o actitudes de una persona vincula a un determinado comportamiento antisocial y la adquisición por el sujeto de tales 
definiciones pro delictivas facilitaran el inicio y el mantenimiento de la conducta delictiva; el reforzamiento diferencial es aquel proceso de balance entre los refuerzos y los castigos anticipados o reales que siguen o son consecuencia de una determinada conducta, en este modelo de condicionamiento operante que establece que las consecuencias que siguen a una conducta son un determinante de la probabilidad futura de que se repita - mediante recompensas sociales o materiales o a través de auto reforzamiento o lenguaje interno de auto gratificación, precipitarían el inicio y el mantenimiento de la conducta antisocial, finalmente la imitación de modelos consiste en involucrarse en alguna conducta después de observar en otros comportamientos semejante, esto dependerá de las características intrínsecas del modelo, de la conducta observada en este y de la capacidad del sujeto para poder reproducir esa conducta adecuadamente y de las consecuencias de ese comportamiento a seguir han generado.

La teoría del modelo ecológico de Bronfenbrenner (1987-1994) citado en Frías et al (2013) propone una perspectiva ecológica del desarrollo de la conducta humana. Desde esta perspectiva, el desarrollo es concebido como un fenómeno de continuidad y cambio de las características biopsicológicas, tanto de los individuos como de los grupos. El modelo incluye la experiencia, que implica no sólo las propiedades objetivas, sino también las que son subjetivamente experimentadas por las personas que viven en un determinado ambiente.

\section{Conclusiones}

Los factores de riesgo personales que presentan los adolescentes infractores del centro juvenil de el Tambo, son actitudes que no corresponden para su edad, hecho que ha ocasionado problemas, peleas con sus amigos o compañeros, presentan conductas poco tolerables, no respetan las opiniones o decisiones de los demás, presentan dificultades para resolver sus problemas, muchos han sido agredidos por sus compañeros y presentan una débil autoestima.

En los factores de riesgo en los escolar que presentan los adolescentes infractores del centro juvenil de el Tambo son el registro de faltas en sus colegios, presentaron problemas con sus compañeros del colegio, incumplimiento de normas establecidas en el colegio, hechos que generó sanciones, negatividad en la participación de actividades institucionales del colegio, incumplimiento de tareas académicas, problemas con sus profesores, evasión al colegio, asignaturas desaprobadas y el consumo de licor o drogas.

Con respecto a los factores de riesgo en lo familiar que presentan los adolescentes infractores del centro juvenil de el Tambo, padres de familia que han sido detenidos o recluidos en algún establecimiento penitenciario, haber recibido castigos por parte de sus padres cuando eran niños, evidenciaron situaciones de peleas entre sus padres por cuestiones económicas.

Finalmente concluimos en la investigación que los factores de riesgo medio ambiental social que presentan los adolescentes infractores del centro juvenil de El Tambo son los personales, familiares y escolares. 
Referencias bibliográficas

Barraza, R. (2009). Delincuencia juvenil y pandillerismo. Archivos de Criminología,Criminalística y Seguridad Privada, (3), 1-10.

Farrinton, D. (1992) Explaining the beginning. Progress and ending of antisocial behavior from bith to adulthoo. En J. McCord (ed) Facts, framework and forocasts. Advance en criminological theory (vol.3) New Brunswick, N.J. transaction publichers.

Flores, C.N. (2018) Factores que inciden en el infractor penal niño y adolescentes en el Distrito Judicial de Lima - Este, escuela de posgrado de la Universidad César Vallejo, Lima Perú. http://repositorio.ucv.edu.pe/bitstream/handle/UCV/19426/Flores_ CN.pdf?sequence $=1 \&$ is Allowed $=y$

Frías, M., López, S. \& Díaz, G. (2003). Predictores de la conducta antisocial juvenil: un modelo ecológico. Estudios de Psicología, 8, 15-24. https://www.scielo.br/scielo.php?pid=S1413294 X2003000100003\&script=sci_arttext

INEI (2015) Informe anual del Instituto Nacional de Estadística e Informática https://www.inei. gob.pe/media/MenuRecursivo/publicaciones_digitales/Est/Lib1446/libro.pdf

INDAGA (2016) Boletin II, Consejo Nacional de Política Criminal ¿Cómo son los adolescentes infractores en el Perú? Ministerio de Justicia y Derechos Humanos. https://img.Ipderecho. $\mathrm{pe} / \mathrm{wp}$-content/uploads/2016/11/MINJUS-C\%C3\%B3mo-son-los-adolescentes-infractoresen-el-Per\%C $3 \%$ BA.pdf

López, S. \& Rodríguez, J. (2012) Factores de riesgo y de protección en el consumo de drogas y la conducta antisocial en adolescentes españoles, Universidad de Coruña, España. https://www. redalyc.org/pdf/2990/299023539004.pdf

Redondo, S., Andrés - Pueyo, A. y Catena, A. (2011). Factores de éxito asociados a los programas de intervención con menores infractores. Informes, estudios e Investigación, Ministerio de sanidad, política social e igualdad. https://www.observatoriodelainfancia.es/oia/esp/ documentos_ficha.aspx?id=3687

Redondo, S. (2008) Individuos, sociedades y oportunidades en la explicación y prevención del delito: modelo del triple riesgo (TRD). Revista Española de Investigación criminología.

Sanabria, A. \& Uribe (2010) Factores psicosociales de riesgo asociado a conductas problemáticas en jóvenes infractores y no infractores, Pontificia Universidad javeriana de Colombia, Universidad Pontificia Bolivariana Bucaramanga, Colombia. https://www.redalyc.org/ pdf/679/67915140005.pdf

Sanchez, K.y Velasquéz, L. (2016) Análisis de los factores de riesgo que influyen en los adolescentes infractores de la ley penal en Girardot, Cundinamarca, Corporación Universitaria minuto de Dios, Trabajo Social Girardot, Cundimarca. https://repository.uniminuto.edu/bitstream/ handle/10656/4809/T.TS\%20SANCHEZ\%20GRISALES\%20KELLY\%20NATALIA\%202016. pdf? sequence $=1$ \&isAllowed $=y$

Sierra, S. (2019) Satisfacción familiar y conductas disociales en estudiantes del 5to. Año de secundaria de las instituciones Educativas Francisco Antonio de Zela y Coronel Bolognesi - Tacna, 2018. http://repositorio.upt.edu.pe/bitstream/UPT/946/1/Sierra-Papel-Shelmy.pdf

Valariño, Amado, Alves (2013) Menores infractores: un estudio de campo de los factores de riesgo, https://www.researchgate.net/publication/256648787_Menores_infractores_un_estudio_de_ campo_de_los_factores_de_riesgo_Juvenile_offenders_A_field_study_of_the_risk_factors

Vásquez, G.C. (2015) Predicción y prevención de la delincuencia juvenil según las teorías del desarrollo social https://adolescenciaantisocial.blogspot.com/2015/04/prediccion-yprevencion-de-la.html 